\title{
Metodologia da problematização e o desenvolvimento de habilidades interpessoais na formação profissional em saúde
}

\author{
Problematization methodology and the development of interpersonal skills in professional \\ training in health
}

\author{
J. S. Rodrigues ${ }^{1 *}$, I. M. Souza ${ }^{2}$, J. C. Souza ${ }^{3}$, M. C. Souza ${ }^{4}$ \\ ${ }^{1}$ Departamento de Medicina de Lagarto, Universidade Federal de Sergipe, Campus prof. Antonio Garcia \\ Filho,49400-000, Lagarto-SE, Brasil. \\ ${ }^{2}$ Departamento de Educação em Saúde de Lagarto, Universidade Federal de Sergipe, Campus prof. Antonio Garcia \\ Filho, 49400-000, Lagarto-SE, Brasil. \\ ${ }^{3}$ Departamento de Enfermagem de Lagarto, Universidade Federal de Sergipe, Campus prof. Antonio Garcia Filho, \\ 49400-000, Lagarto-SE, Brasil. \\ ${ }^{4}$ Universidade Luterana do Brasil, Canoas-RS, Brasil. \\ *mylle_sr@hotmail.com
}

(Recebido em 30 de outubro de 2016; aceito em 19 de abril de 2017)

\begin{abstract}
O presente trabalho objetiva demonstrar a importância da Metodologia da Problematização, adotada pela Universidade Federal de Sergipe, campus de Lagarto/SE, para a graduação em cursos da área da saúde, sendo esta metodologia utilizada pela disciplina de Práticas de Ensino na Comunidade. Pretende-se ressaltar o desenvolvimento das habilidades de relacionamento interpessoal, empatia, crítica, apreensão da realidade, iniciativa, comunicação e trabalho em equipe. Características essas, que são fundamentais para a sociedade atual, que convive com aceleradas modificações, acúmulo de informações e perda gradativa da habilidade de compreender a subjetividade das pessoas. Para tanto, será apresentado um relato de experiência do conjunto de atividades desenvolvidas durante o ano letivo de 2014-2015 pelos discentes de quatro dentre os oito cursos do campus (Enfermagem, Farmácia, Fisioterapia, Fonoaudiologia, Medicina, Nutrição, Odontologia e Terapia Ocupacional), com enfoque em uma turma específica, bem como dos aspectos que permearam esta prática. Do mesmo modo, são explicitados pontos fundamentais para o tema, como a definição da metodologia da problematização, o conceito de saúde, contribuições de Paulo Freire e a Política Nacional de Humanização.

Palavras-chave: saúde, habilidades socioemocionais, formação, humanização, metodologia da problematização.
\end{abstract}

This paper aims to demonstrate the importance of the Problematization Methodology, adopted by the Federal University of Sergipe, campus Lagarto / SE, for graduation in health area courses, and the methodology used by the discipline of Teaching Practices in the Community. It is intended to emphasize the development of interpersonal skills, empathy, critical apprehension of reality, initiative, communication and teamwork. These features, which are fundamental to modern society, living with accelerated changes, accumulation of information and gradual loss of ability to understand the subjectivity of people. To do so, will be presented a set of experience report of activities undertaken during the academic year 2014-2015 by students of four of the eight campus courses (Nursing, Pharmacy, Physical Therapy, Speech Therapy, Medicine, Nutrition, Dentistry and Occupational Therapy), focusing on a specific class, as well as the aspects that permeate this practice. Similarly, are detailed key points to the theme, such as the definition of the problematic methodology, the concept of health, Paulo Freire contributions and the National Humanization Policy.

Keywords: health, social-emotional skills, training, humanization, problematization methodology. 


\section{INTRODUÇÃO}

O campus professor Antonio Garcia Filho, da Universidade Federal de Sergipe (UFS), localizado em Lagarto SE, utiliza metodologias ativas em todas as grades curriculares dos oito cursos que oferece. Os alunos de Enfermagem, Farmácia, Fisioterapia, Fonoaudiologia, Medicina, Nutrição, Odontologia e Terapia Ocupacional têm aulas juntos em todas as disciplinas do primeiro ano, mas especialmente na disciplina de Práticas de Ensino na Comunidade (PEC), eles são instigados a compreender o processo saúde-doença de modo holístico e a buscar soluções para os problemas da realidade.

De um modo geral, as metodologias ativas consistem em métodos de ensino que vêm sendo incluídos em diversos cursos superiores, especialmente os da área da saúde. Como exemplos de metodologias ativas aplicadas ao ensino superior, temos o estudo de caso, o processo do incidente, método de projetos, aprendizagem baseada em problemas e a metodologia da problematização. Os cursos da UFS de Lagarto possuem três grupos de disciplinas (que neste campus são denominadas subunidades): tutoriais (que englobam diversos conteúdos), habilidades (aulas práticas e estágios) e PEC (alia o ensino com a comunidade e os serviços de saúde). A aprendizagem baseada em problemas é utilizada nas sessões tutoriais e habilidades mescla diversas metodologias.

A PEC utiliza a metodologia da problematização, a qual consiste em uma estratégia de ensino-aprendizagem que proporciona aos estudantes da saúde a sedimentação teórico-prática dos conteúdos através do processo ativo de construção do conhecimento [1][2]. Além disso, ela possibilita o desenvolvimento de habilidades socioemocionais de trabalho em equipe e de relacionamento com os usuários do serviço de saúde, sendo que este ocorre através da compreensão da realidade das pessoas, bem como dos determinantes socioeconômicos, políticos e culturais que interferem na qualidade de vida.

Berbel [1], ao tratar da problematização, define esta estratégia de ensino como um processo no qual a participação ativa na solução de problemas reais, o diálogo entre alunos e professores e a estimulação do potencial dos discentes é importante para a sua formação integral. Para tanto, ela cita o Método do Arco de Charlez Marguerez, segundo o qual partindo da realidade, os estudantes observam os seus problemas, elencam pontos-chave, procuram informações por meio da teorização, elaboram hipóteses de solução e por último efetuam a aplicação à realidade.

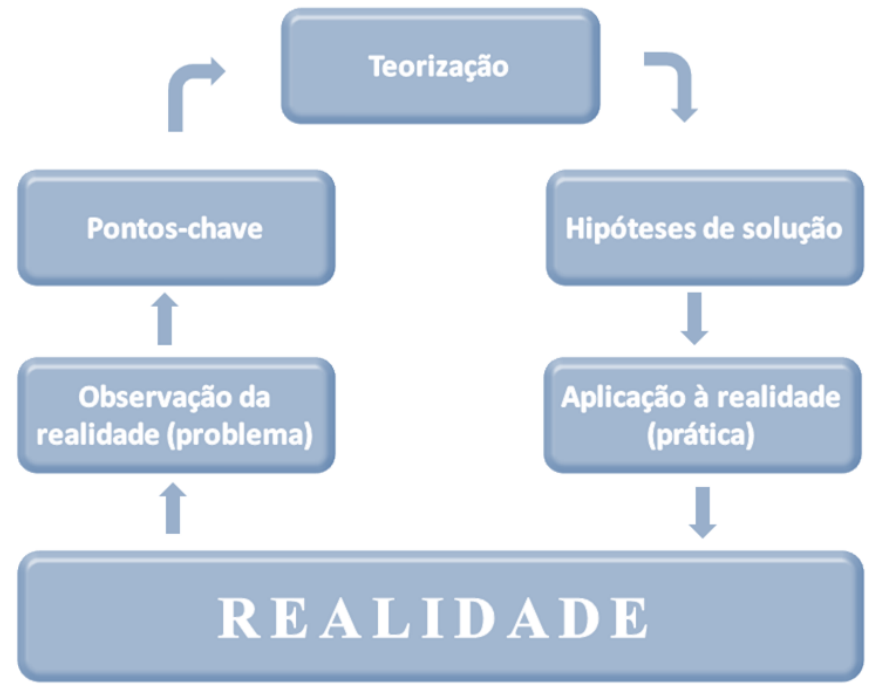

Figura 1 Arco de Maguerez, segundo Berbel, a partir de Bordenave e Pereira

Igualmente é abordado por Berbel que, para a promoção da autonomia dos estudantes, é necessário que haja envolvimento pessoal, baixa pressão, alta flexibilidade em sua execução, percepção de liberdade psicológica e de escolha. Essas ações visam evitar que os estudantes se reconheçam como "marionetes", o que provoca sentimentos de fraqueza, ineficácia, desmotivação e desvinculação com os eventos do ambiente. 
Este método também é importante para superar o que Paulo Freire define como educação bancária, caracterizada por ser fundamentalmente narradora e dissertava. Em sua obra, Pedagogia do Oprimido, ele relata que "falar da realidade como algo parado, estático, compartimentado e bem comportado" ou abordar "algo completamente alheio à experiência existencial dos educandos vem sendo, realmente, a suprema inquietação desta educação" [8]. Portanto, em todas as áreas da educação, mas singularmente para a formação saúde, essa reflexão torna-se essencial.

A saúde é definida pela OMS (Organização Mundial de Saúde) como "o estado do mais completo bem-estar físico, mental e social e não apenas a ausência de enfermidade" [14], o qual ressalta a importância dos aspectos mentais e sociais na saúde das pessoas. Em 2003, ao longo dos 15 anos de existência do SUS (Sistema Único de Saúde), notou-se, através da avaliação dos serviços, "o despreparo de profissionais para lidar com a dimensão subjetiva que toda prática de saúde supõe" [10].

Então, em 2003, o Ministério da Saúde instituiu o Programa Nacional de Humanização - PNH em Saúde, pois mesmo com o desenvolvimento de novas técnicas, diagnósticos, aquisição de conhecimentos na área da saúde, "não se verificava o aperfeiçoamento das práticas de saúde, como práticas compostas pela comunicação, pela observação, pelo trabalho de equipe, por atitudes fundamentadas em valores humanitários sólidos" [6]. Essa política pressupõe vários eixos de atuação, sendo que no eixo da educação permanente, é declarado que a PNH deve ser incluída como conteúdo ou componente curricular dos cursos de graduação em saúde [10][11].

Aliando a problematização aos cursos da saúde, os estudantes podem ter um contato efetivo com a realidade, que passa a ser vista não somente através de aspectos conceituais da prática do cuidado à saúde, mas também os aspectos sociais e políticos da realidade. Além disso, a metodologia também representa um desafio para os docentes, que são fundamentais para que o método tenha efetividade.

\section{METODOLOGIA}

O desenvolvimento das atividades de PEC ocorreu nas unidades básicas de saúde do município de Lagarto/SE, localizadas em áreas centrais, periféricas ou nos povoados. As ações foram fundamentadas na Metodologia da Problematização, representada pelo Arco de Maguerez, e em artigos sobre determinantes sociais em saúde, cidadania, participação popular, epidemiologia, saúde coletiva, promoção da saúde, atenção básica, atenção primária, aprendizagem baseada em problemas, territorialização, bem como também foram estudadas as leis 8080/90 (que dispõe sobre a organização e funcionamento do SUS) e a lei 8142/90 (que trata da participação popular, um dos princípios do SUS).

As ações foram desenvolvidas durante o ano letivo de 2014-2015 foram realizados em diversas turmas contendo entre nove e catorze discentes, buscando mesclar alunos de pelo menos quatro cursos diferentes. $\mathrm{O}$ relato a seguir corresponde às vivências e práticas realizadas pela turma 15 , composta por discentes de Enfermagem, Farmácia, Fisioterapia e Medicina, que ocorreram na Unidade Básica de Saúde (UBS) Davi Marcos de Lima, localizada no bairro Jardim Campo Novo em Lagarto-SE, bem como na comunidade em seu entorno.

Inicialmente, procedeu-se à análise, reconhecimento do território e dos determinantes sociais de saúde da população local. Foram realizadas inúmeras visitas e inquéritos comunitários sobre concepções de saúde e necessidades em saúde, além de outras atividades como: acompanhamento do trabalho dos agentes comunitários de saúde; observação e discussão em sala de espera da unidade; conhecimento das Redes de Atenção à Saúde (RAS) do município, como o Centro de Atendimento Psicossocial (CAPS), Conselho tutelar e a Associação dos Pais e Amigos dos Excepcionais (APAE), dentre outros órgãos; entrevista com um dos médicos da UBS.

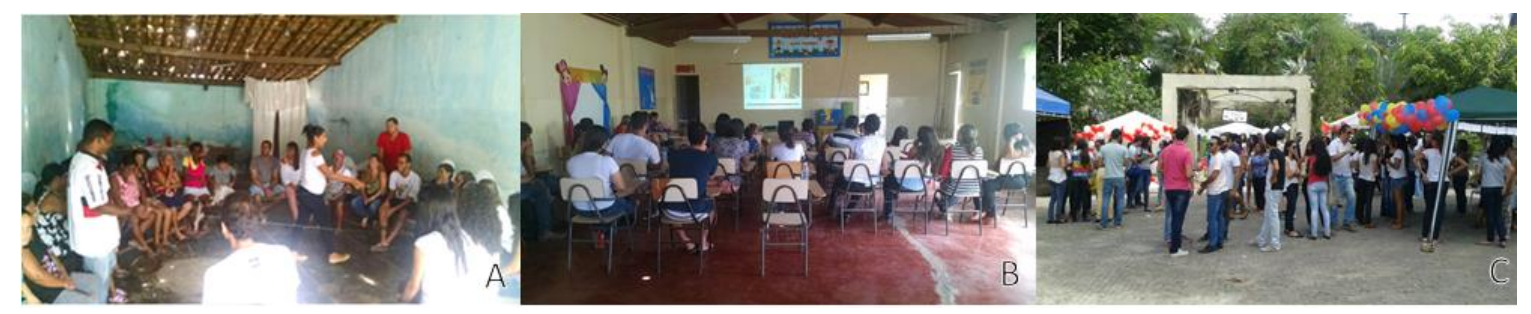


Figura 2: Ações na comunidade. A e B: Ações no bairro Jardim Campo Novo. C: Mostra de experiências na Praça Filomeno Hora

Foi promovida uma roda de conversa com a população sobre saúde, direito e cidadania e elaborado um documentário com os moradores relatando as suas condições de vida, o qual foi socializado com a comunidade. As ações foram finalizadas com a mostra de experiências das atividades desenvolvidas por todas as turmas do primeiro ano na disciplina de PEC, ao final do ano letivo 2014-2015 para a população de Lagarto/SE na Praça Filomeno Hora, um dos locais onde os movimentos culturais do município costumam se apresentar.

Em virtude de captar a experiência pessoal que cada integrante da turma teve com as vivências desta subunidade, foi aplicada uma pesquisa do tipo levantamento, no formato de um questionário. $\mathrm{O}$ instrumento de pesquisa escolhido foi aplicado através da plataforma Formulários Google e os dados foram modificados pelo programa Microsoft Word 2010. Além disso, os participantes foram devidamente informados do que se tratava a pesquisa e declararam conhecimento acerca da resolução CNS n ${ }^{\circ} 466$, de 12 de dezembro de 2012 [5], que trata da pesquisa com seres humanos.

O questionário continha 09 perguntas, sendo que as cinco primeiras tinham três opções "discordo", "não concordo, nem discordo" e "concordo". Havia também uma questão objetiva para que o entrevistado (a) classificasse a sua experiência pessoal com PEC como "ruim", "regular" ou "boa". Dentre as três perguntas restantes, uma era discursiva na qual o (a) entrevistado (a) poderia acrescentar alguma informação ou experiência vivida, a penúltima se referia à identificação do curso ao qual pertence e a última era para declarar o conhecimento acerca do que a pesquisa aborda.

\section{RESULTADOS E DISCUSSÃO}

Dentre os 14 participantes da turma 15 de Práticas de Ensino na Comunidade, 08 responderam ao questionário, o que representa 57\% dos integrantes. As opções eram objetivas em uma escala linear de 01 a 03 em que nas cinco primeiras perguntas 01 correspondia a discordar e 03 correspondia a concordar. Já em relação aos cursos das pessoas entrevistadas, havia 04 discentes de enfermagem, 02 de farmácia, 01 de fisioterapia e 05 de medicina.

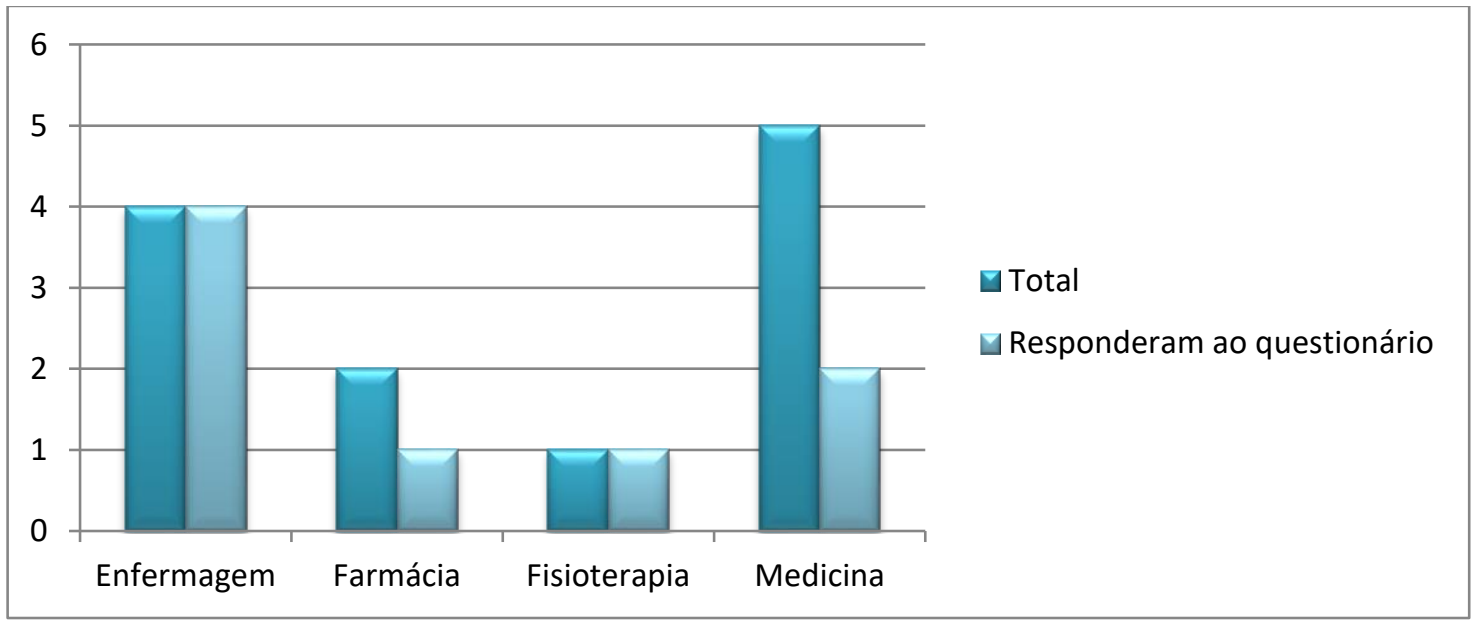

Figura 2 Gráfico comparativo com a quantidade de entrevistados

Dentre os entrevistados, $05(87,5 \%)$ concordaram que a Metodologia da Problematização os fizeram repensar o modo como interpretam o mundo, a mesma porcentagem concordou que hoje compreendem a importância dos determinantes sociais da saúde no modo como a sociedade está hoje estruturada, um (a) discente do curso de farmácia marcou uma resposta neutra (não discordou, nem concordou). Após a terceira e quarta indagações, 05 (87,5\%) concordaram que ter conhecimento das leis que regem o SUS é importante para a futura atuação profissional e de que conhecer um pouco da atuação das redes de atenção em saúde os fizeram ampliar seus conceitos sobre a abrangência da saúde, um (a) discente de fisioterapia marcou uma resposta neutra. 
Além disso, 05 (87,5\%) entrevistados concordaram que elaborar e executar uma ação de intervenção na comunidade juntamente com os colegas os ajudaram a trabalhar em equipe e a pensarem em estratégias para solucionar problemas reais, um (a) discente de medicina marcou uma resposta neutra. A sexta pergunta, para que o (a) entrevistado qualificasse a experiência pessoal que teve com a PEC, 05 (62,5\%) definiram como "boa", e 03 (37,5\%) como "regular", sendo que estes 03 discentes pertencem aos cursos de enfermagem, farmácia e fisioterapia.

A maioria dos entrevistados, não respondeu a pergunta discursiva, entretanto, 02 discentes de enfermagem e 01 de medicina expuseram seus depoimentos:

A PEC do primeiro ciclo ampliou minha visão sobre a atual situação da cidade em que vivo, e me ofereceu uma nova forma de fazer a diferença.

A PEC mudou bruscamente a minha visão sobre o SUS, antes eu só o via como algo negativo e problemático, durante e após a PEC I, passei a ter outra concepção sobre o sistema único de saúde, como algo que ainda tem muito o que melhorar, mas que é imprescindivel para o processo saúdeldoença da população brasileira.

Cursar a disciplina de PEC do primeiro ano me fez confrontar com os aspectos biopsicossociais e políticos que interferem de modo determinante na saúde. Muitos problemas de saúde decorrem destas condições mais complexas que na maioria das situações nós, futuros profissionais de saúde, não poderemos intervir de modo resolutivo. Porém, devemos fazer o tudo o que for possível para tornar a qualidade de vida das pessoas a melhor possível.

Os resultados da pesquisa mostraram que a metodologia aplicada com esta disciplina não foi proveitosa para todos de modo unânime e que não há como correlacionar este achado com o curso que a pessoa está fazendo. Porém, de um modo geral, percebe-se que a metodologia da problematização produziu um impacto positivo na vida da maioria dos participantes da amostra em questão e que possui grande potencial de aprimoramento para a formação dos futuros profissionais de saúde. Este desafio foi exposto por Sobral e Campos (2012) [15] em uma revisão bibliográfica que explicita os obstáculos que se interpõem ao emprego da problematização como um novo método de ensino.

Concomitantemente, a pesquisa também demonstrou que a abordagem da subunidade também produz impactos positivos o que é corroborado por Villardi, Cyrino e Berbel (2011) [16], Prado et al (2012) [13] e Maia (2014) [9].

\section{CONCLUSÃO}

A atuação junto à comunidade permitiu aliar a teoria e a prática, condensando a primeira em experiências significativas e reais. Os discentes puderam vivenciar a falta de saneamento básico, de perspectiva de melhoria, o descaso governamental, bem como críticas e elogios a unidade de saúde do bairro. Pôde também ser evidenciado o quanto a carência da educação e de sua valorização torna as pessoas vulneráveis a condições menos favoráveis em relação a sua saúde.

As vivências e práticas na comunidade proporcionaram uma maior compreensão sobre o processo saúde-doença, os determinantes sociais da saúde, as políticas públicas de saúde, bem como, a prática da empatia, a qual "envolve um sentimento de sensibilização pelas mudanças sentidas e refletidas, momento a momento, pela outra pessoa" [7].

Igualmente, buscou-se desenvolver habilidades de relacionamento interpessoal para que se evite o modelo biomédico, que está centrado somente na doença, deixando em segundo plano a educação e a promoção da saúde [12], e para que aja uma boa integração da equipe multiprofissional de saúde, resultando em benefícios para a saúde coletiva. Então, a formação problematizadora de PEC objetiva capacitar os futuros profissionais da saúde para, além de terem conhecimento técnico-científico para enfrentamento das doenças, serem participantes ativos na resolução dos problemas vivenciados no cotidiano, tendo a consciência de que o elemento principal é o ser humano. 


\section{REFERÊNCIAS}

1. Berbel NAV. As metodologias ativas e a promoção da autonomia de estudantes. Semina: Ciências Sociais e Humanas. 2011;(1):25-40.

2. Berbel NAV, Gamboa SAS. A metodologia da problematização com o Arco de Maguerez uma perspectiva teórica e epistemológica. Filosofia e Educação. 2012;3(2).

3. Brasil. Lei $\mathrm{n}^{\mathbf{0}}$ 8.080, de 19 de setembro de 1990. Disponível em: <http://www.planalto.gov.br/ccivil_03/leis/18080.htm> Acesso em 17 jan 2016.

4. Lei $\mathrm{n}^{\mathrm{o}} 8.142$, de 28 de dezembro de 1990. Disponível em: <http://www.planalto.gov.br/ccivil_03/leis/18142.htm> Acesso em 17 jan 2016.

5. Resolução CNS $\mathrm{n}^{\circ} 466$, de 12 de dezembro de 2012. Disponível em: <http://conselho.saude.gov.br/resolucoes/2012/Reso466.pdf> Acesso em 28 jan 2017.

6. Carpara A, Rodrigues J. A relação assimétrica médico-paciente: repensando o vínculo terapêutico. Ciência \& Saúde coletiva. 2004;9(1).

7. Costa FD, Azevedo RCS. Empatia, Relação Médico-paciente e Formação em Medicina: um Olhar Qualitativo. Revista Brasileira De Educação Médica, 2010;34(2):261-269.

8. Freire P. Pedagogia do Oprimido. 50 ed. Rio de Janeiro: Paz e terra, 2011.

9. Maia JA. Metodologias Problematizadoras em Currículos de Graduação médica. Revista Brasileira de educação Médica. 2014;38(4):566-574.

10. Ministério da Saúde. Humaniza SUS - Caderno de Textos - Cartilhas da Política Nacional de Humanização. 2 ed. Brasília: Editora do Ministério da Saúde, 2010.

11. HumanizaSUS: Documento base para gestores e trabalhadores do SUS. 4 ed. Brasília: Editora do Ministério da Saúde, 2008.

12. Pelicioni MCF, Pelicioni AF. Educação e promoção da saúde: uma retrospectiva histórica. O Mundo da Saúde. 2007;31(3):320-328.

13. Prado et al. Arco de Charles Maguerez: refletindo estratégias de metodologia ativa na formação de profissionais de saúde. Escola Anna Nery. 2012;16(1).

14. Scliar M. História do Conceito de Saúde. PHYSIS: Revista de Saúde Coletiva. 2007;17(1):29-41.

15. Sobral FR, Campos CJG. The use of active methodology in nursing care and teaching in national productions: an integrative review. Revista da Escola de Enfermagem da USP. 2012;46(1):202-11.

16. Villardi ML, Cyrino EG, Berbel NAV. A Problematização em Educação em Saúde: percepções dos professores tutores e alunos. 1 ed. São Paulo: Cultura Acadêmica, 2015. 